\title{
When Mechanical Computations Explain Better
}

\author{
Silvano Zipoli Caiani
}

\begin{abstract}
In this paper I defend the epistemic value of the representationalcomputational view of cognition by arguing that it has explanatory merits that cannot be ignored. To this end, I focus on the virtue of a computational explanation of optic ataxia, a disorder characterized by difficulties in executing visually-guided reaching tasks, although ataxic patients do not exhibit any specific disease of the muscular apparatus. I argue that addressing cases of patients who are suffering from optic ataxia by invoking a causal role for internal representations is more effective than merely relying on correlations between bodily and environmental variables. This argument has consequences for the epistemic assessment of radical enactivism, which invokes the Dynamical System Theory as the best tool for explaining cognitive phenomena.
\end{abstract}

Keywords Computational explanation $\cdot$ Dynamical system theory $\cdot$ Radical enactivism $\cdot$ Visual affordances $\cdot$ Optic ataxia

\section{Introduction}

According to a new generation of scholars, the computational paradigm that have informed the study of cognition for decades now creak under the weight of the new enactivist approach to cognition. Over the last few years, indeed, several philosophers and cognitive scientists have proposed to replace the mechanical and representational assumptions underlying the computational paradigm with a dynamical and extensional way to understand cognition (e.g., Chemero, 2011; Hutto \& Myin, 2017, Gallagher, 2017). Supporters of the radical enactivist view (RE) argue that the computational paradigm does not add explanatory power over and above the physical

\footnotetext{
S. Zipoli Caiani ( $\varangle)$

Università Degli Studi Di Firenze, Florence, Italy

e-mail: silvano.zipolicaiani@unifi.it 
description of a cognitive system, and therefore it should be abandoned (e.g., van Gelder, 1995; Chemero, 2011; Hutto \& Myin, 2012). ${ }^{1}$

The aim of this paper is to defend the epistemic value of the computational view of cognition by arguing that it has explanatory merits that cannot be ignored. To this end, I focus on the virtue of a mechanical-computational explanation of the behavior of patients suffering from optic ataxia, a disorder characterized by difficulties in executing visually-guided reaching tasks, although patients do not exhibit any specific disease of the muscular apparatus (Balint, 1909). I argue that addressing cases of patients who are suffering from optic ataxia by invoking the causal role for internal representations is more effective than merely relying on correlations between bodily and environmental variables.

According to the computational paradigm, the cognitive system forms visual representations of the available actionable opportunities in the environment, which have a causal role in action planning and execution (e.g., Fodor \& Pylyshyn, 1988; Mcculloch \& Pitts, 1944; Putnam, 1967). This serves to emphasize the need to identify the parts and the mechanical structures characterizing the causal chains underlying and generating the behavior of interest (Craver \& Darden, 2013; Illari \& Williamson, 2012; Bechtel \& Richardson, 1993; Craver, 2006). Thus, such an account shows why an agent performs a certain behavior by describing the relevant mechanisms linking internal representations with the agent's motor system. ${ }^{2}$

In a different vein, RE denies the need to invoke internal representations to account for the interaction between vision and action. According to RE, modeling the relationships between vision and action requires attending to the ways in which individuals dynamically engage with certain worldly offerings by means of extended interactions (Hutto \& Myin, 2017). In doing this, RE assumes that visual cognition does not involve the selecting, storing, and processing of information in the brain. Differently, $\mathrm{RE}$ conceives visual cognition as an extensive phenomenon concerning the variation of bodily and environmental variables spanning multiple temporal and spatial scales. This amounts to an assumption that the agent and the environment form a unified system whose behavior cannot be modeled as a causal chain linking separate parts (Chemero, 2011). Accordingly, the interlocking between vision and action should be explained via a methodological framework that does not posit mental representations, like dynamical systems theory (DST). Notably, modeling cognition by means of DST allows for a lawful account of how agents interact with the action-related properties of the environment, without the need to involve internal resources such as causal states and computations (e.g., Beer, 2000; Spivey, 2008; Chemero, 2011).

\footnotetext{
${ }^{1}$ For the sake of the present argument, I focus exclusively on Radical Enactivism (e.g., Chemero, 2011; Hutto \& Myin, 2012), excluding the different theoretical strands that populate the enactivist world (e.g., Maturana \& Varela, 1991; Noë, 2004; O'Regan \& Noë, 2001; O’Regan, 2011). At present, radical enactivism is the most developed, discussed, and challenging alternative to the classical computational paradigm that has informed cognitive science for about sixty years.

${ }^{2}$ It should be noted that the mechanical approach to explanation improves our comprehension of the causal chain that allows a behavior to occur in conjunction with certain environmental conditions, thus making the execution of an action a non-surprising event (Cohen, 2015; Schupbach \& Sprenger, 2011).
} 
Though RE is currently encountering enthusiastic appraisals, it is not un-common that someone may still consider it as a proposal that is more easily explained than proved. Whether RE is only on the crest of a fashionable wave that is doomed to leave no tracks in the sand, or whether it is a tsunami with the power to sweep away the existing explanatory practice in the cognitive sciences, is something that has not yet been carefully assessed. In order to address this issue, I follow Hutto and Myin in considering that the only naturalistically respectable way to decline RE is "to give it its day in empirical court" (Hutto \& Myin, 2017, p. 19). This amounts to wondering whether the methodological tools of DST, instead of a computational-mechanical approach, offer the best explanation of basic cognitive phenomena. This paper shows that there are factual circumstances concerning the ability and inability to perceive and exploit visual affordances for which DST is not able to explain, but which are suitably accounted for through the adoption of a computational architecture based on the dual streams model of vision (Goodale \& Milner, 1992). More precisely, I maintain that RE provides a valuable explanation of why agents perceive action opportunities, whereas a computational view provides an explanation for why agents have such an ability in addition to why this ability can be lost.

This paper is divided into six parts. In the first part (Sect. 2), I introduce RE by distinguishing between two claims, the former concerning the ontological status of representational entities, and the latter concerning the explanatory power of a nonrepresentational account of cognition. In Sect. 3, I focus on the explanatory claim and provide details concerning the strategy underlying DST, showing that it amounts to a correlational approach. In Sect. 4, I introduce the case of optic ataxia, and argue that it is an ideal target for measuring the explanatory power of DST. In Sect. 5, I show that the correlational analysis provided by DST is not suitable to explain relevant aspects of ataxic behavior, since it does not suffice to provide an etiological account for it. Finally, in Sect. 6, I introduce a computational model of vision for action, and show that it is suitable to provide the etiological account that is required in the case of optic ataxia.

As a result, although sometimes the dynamical systems theory and the computational paradigm can be "natural allies", playing both a complementary role in describing the interactions between vision and action (Kaplan, 2015), in the case of optic ataxia, the computational view is more explanatory than the dynamical one. This outlines that there is an epistemological shortcoming of radical enactivism compared to the computational account.

\section{Radical Enactivism and the Explanatory Claim}

According to RE, there are cognitive facts that can be fully and completely accounted for by means of an extensional language, that is, by conceiving them merely in terms of activities in which the agent's body is dynamically engaged with the environment. Notably, considering cognitive phenomena in a purely extensional way, supporters 
of RE state that the body-environment relations do not involve any computational manipulation of information (e.g., Chemero, 2011; Hutto \& Myin, 2012, 2017).

In denying the computational nature of cognition, supporters of RE might be committed to more than one claim. As Chemero (2011) has noted, when one proclaims that cognition does not involve computations, there are at least two theoretical views one might endorse. First, one might be making a claim about what there is and what there is not, namely, a claim about the ontology of the cognitive sciences. Second, one might be claiming something about the best way to provide explanatory arguments in the cognitive sciences. While in the former case, the rebuttal of the computational view amounts to a metaphysical thesis, in the latter case it rests on epistemological grounds, that is, on the analysis of the needs and practices that characterize the work of cognitive scientists. The key difference between the two claims is that only the explanatory claim is an empirical hypothesis, whereas the metaphysical claim concerns our philosophical criteria for establishing the place of cognition in nature (Chemero, 2011).

Over the last decades, many arguments have been raised against the attempt to provide a successful naturalization of computational systems (for a review see, Kriegel, 2013; Pietroski, 1992; Ramsey, 2007, 2015), such that it is an ongoing debate whether computational processes should be considered parts of the natural ontology or not. Although it raises a fascinating philosophical discussion, the metaphysical hypothesis has little impact on the scientific practice since one may continue to refer to a computational approach to cognition with or without compromising with any sort of naturalization of the computational states (classically Dennett, 1987; more recently see Egan, 2013; Colombo, 2014). Accordingly, given the different purposes underlying the practical use of a word such as "computations", the metaphysical claim is hardly defensible on empirical grounds (Chemero, 2011).

Differently, the epistemological hypothesis concerning the explanatory value of the computational approach to cognition has dramatic consequences on the real practices of cognitive scientists. According to this hypothesis, the great variety of experiences and behaviors are best understood without appealing to the manipulation of causal states but rather by focusing on the dynamical interactions between the agent's body and the environment. When it comes to accounting for intelligent activity, supporters of RE subscribe to the Equal Partner Principle (Hutto \& Myin, 2017), according to which variables of any kind make an equal explanatory contribution, regardless of whether they concern aspects located in or out of the boundaries of skull and skin. This means that citing internal factors endowed with a causal status does not carry more explanatory value than, for example, referring to environmental and bodily factors that merely correlate with each other. Accordingly, since the computational view is refuted as an explanatory tool for the cognitive sciences, agents and environmental factors can be modeled as a unified, non-decomposable system whose behavior cannot be accounted for, even approximately, as a set of separate causal parts. 


\section{Radical Enactivism and the Dynamical System Theory}

According to the previous considerations, the adoption of DST may be pivotal for an epistemological approach to RE (e.g., Beer, 2000; Chemero, 2011; van Gelder, 1995; Heinke, 2000; Walmsley, 2008). Indeed, the methodological assumptions underlying DST allow for an approach to the study of cognition that avoids mechanical states and inner computational processing (Spivey, 2008, Chemero, 2011). Conceiving cognition from an extensional point of view allows for a lawful account of how agents interact with the action-related properties of the environment, without the need to involve internal resources such as causal states and computations. According to DST, cognitive explanations are arguments based on factual premises and inferential rules inasmuch as they take the form of a reasoning in which the phenomenon to explain (explanandum) follows as a deductive consequence of the selected premises (explanans). This is, indeed, the core idea of the well-known covering-law model of explanation (Hempel, 1965; Walmsley, 2008). ${ }^{3}$

Over the last few decades, this methodological approach has been endorsed in the cognitive science of vision to account for the way agents perceive an affordance in the environment, that is, a possibility of action that surround the agent's body (Gibson, 1979). According to this view, the perception of affordances is construed as the detection of a relation between features of the environment and certain motorrelated properties of the agent's body. Hence, in order to study the perception of affordances by means of DST, some environmental parameters should be considered in relation to some relevant variables concerning the agent's body and the related motor skills (e.g., Harrison, Turvey, \& Frank, 2016; Lopresti-Goodman, Turvey, \& Frank, 2011; Mark, 1987; Rietveld \& Kiverstein, 2014).

Therefore, if a cognitive agent guides its activity by detecting affordances in the environment, it is possible to suppose that these affordances must be sensible with regard to the lawful relationships between environmental aspects and the relevant features and motor skills of its own body. DST, indeed, starts by selecting the critical parameters that characterize the state of the agent-environment system and attempts to disclose the way such parameters relate with one another. Then, DST focuses on the trajectories in a phase space that the parameters of the agent-environment system traverses, given the covariation of bodily, practical and environmental variables, describing the laws according to which its behavior changes because of the modification of one or more parameters (Beer, 2000; Chemero, 2011).

To this extent, DST improves our access to the laws governing the interactions between the agent's body and its environment, thus making the occurrence of a certain agent's intelligent behavior not a surprising event. This would be particularly evident if we were interested in making predictions concerning the manner in which agents'

\footnotetext{
${ }^{3}$ In this view, one explains the occurrence of a certain event $\mathrm{E}$ by arguing that it is expected because of the factual conditions $C_{1} \ldots C_{n}$ and the deductive laws $L_{1} \ldots L_{n}$. Such a type of explanation is suitable to answer the question "Why does phenomenon E occur?" by showing that its occurrenceor its probability of occurring - results from the combination of particular circumstances $\left(C_{1} \ldots C_{n}\right)$, in accordance with the general laws $\left(\mathrm{L}_{1}, \ldots \mathrm{L}_{\mathrm{n}}\right)$.
} 
behavior varies over time. Indeed, once we know the relevant ambient parameters and the laws governing the dynamical evolution of the environment-agent system, the future values of the agent's behavior become nothing but a matter of deduction. According to this view, if a dynamical systems account is sufficiently accurate to describe what would occur in counterfactual circumstances, it can be considered as a tool suitable for reducing surprise about the occurrence of a behavioral event (e.g., Chemero \& Silberstein, 2008; Thelen \& Smith, 1996).

To sum up, the epistemological approach to RE and the methodological tools of DST form a joint venture that has recently attracted the attention of an increasing number of cognitive scientists. DST, indeed, rests on the Equal Partner Principle by providing an account of the agent's behavior that does not discriminate between internal and external resources. Accordingly, DST offers deductive-nomological explanations that are merely based on the fine-grained analysis of the internal dynamics characterizing the covariation of selected parameters spanning the agent's brain, the body and its environment.

Although RE is gaining an increasing consensus, it is still an open issue whether it will be able to replace the mechanical-computational paradigm that has guided the cognitive sciences over the last sixty years. If so, DST should be able to provide a satisfactory explanation of any sort of cognitive phenomena, with emphasis on the agent's basic cognitive behaviors, such as the perception and misperception of affordances in the environment (Hutto \& Myin, 2012). However, in the remaining part of this paper, I will show that this is not the case.

\section{Explaining Anomalies: The Case of Optic Ataxia}

The study of cognition is not a mere theoretical game, but it has relevant practical implications for the development of therapies and rehabilitation programs for patients suffering from cognitive deficits. Considering this purpose, it is interesting to assess the explanatory virtue of RE as it pertains to its possible clinical consequences. Thus, it may be helpful to assess the adoption of DST as a methodological tool for the explanation of non-standard cases of perception such as optic ataxia, a condition in which some or all aspects of visual guidance of reaching with the hand and arm are lost. Patients suffering from optic ataxia have an intact visual field, good oculomotor control, and normal motor skills; however, they are not able to detect the possible practical relations between their motor abilities and the features of the environment, meaning that they are not able to perceive the affordances available to them.

The scientific literature concerning cases of optic ataxia reports alterations in the initial and final stages of the visually guided movement of reaching to grasp. Anomalous dynamics have been reported in scaling the aperture of the hand according to the target (Cavina-Pratesi, Connolly, \& Milner, 2013) in following objects' trajectories and in executing the final stage of a grasping action (Blangero et al., 2010). Furthermore, ataxic patients show a lack of automatic correction when a target changes location (Pisella et al., 2000) and a lack of ability to avoid collisions with distractors 
when reaching for something (Schindler et al., 2004). However, although optic ataxia is a permanent impairment, patients can relieve their deficit and improve their performances by means of specific rehabilitation programs. For example, patients exhibit an enhanced performance in reaching and grasping when a delay is introduced between the perceptual stimulus and the behavioral response (Himmelbach \& Karnath, 2005). Moreover, a common rehabilitation program includes compensatory strategies such as the recourse to external prostheses (e.g., planners, calendars, recording devices, timers and pagers) in addition to internal cueing (e.g., developing mnemonics or an internal checklist). Generally, patients have been demonstrated to reduce errors and improve performance by following non-perceptual cues, such as conceptual information, but only when their memory is relatively preserved (Zgaljardic et al., 2011).

Evidence such as this begs for an explanation. Notably, two main questions arise: the first concerns the very etiology of optic ataxia, and the second concerns the fact that, at least in certain cases, ataxic patients exhibit good performance. It is interesting, indeed, to understand why patients with lesions precisely located in the parietal cortex are not able to detect and select affordances in the environment and why precisely the execution of delayed tasks and the retrieval of conceptual information improve patient performances (Himmelbach \& Karnath, 2005; Zgaljardic et al., 2011). To this extent, explaining optic ataxia may be used as a testing ground for examining the epistemic virtue of DST. It seems reasonable, indeed, to assume that a good account of basic cognitive abilities should be able to address anomalous cases as well. Accordingly, a valuable explanation of affordance perception should explain why agents may lose such an ability as well as why they may be able to recover it given certain circumstances.

\section{Covariation Is not Enough}

Because DST approaches the perception of the affordances by means of coveringlaw explanations (see Sect. 3), it provides an account of optic ataxia that is addressed on the covariation of selected parameters that characterize the state of the agentenvironment system. Notably, in explaining the anomalous behavior of patients suffering from optic ataxia, DST focuses on the trajectories in a mathematical phase space that the agent-environment system traverses over time, and it specifies how they depend on changes in one or more parameters of the coupled system.

The efforts of scholars working in the context of DST has been merely devoted to observe how patterns of correlation between bodily and environmental variables emerge, stabilize and are sometimes lost. Indeed, according to a correlational approach, explaining anomalous performances in perceiving and exploiting affordances requires the identification of appropriate patterns of variables to quantify and qualify the nature of the deficit. Although DST is usually focused on nondisabled individuals, several studies have recently measured the ability to perform visually guided reaching actions in patients with lesions to the parietal cortex that are 
comparable to those characteristics of optic ataxia (e.g., Kamper et al., 2002; Pisella, Rossetti, \& Rode, 2017). ${ }^{4}$

Correlational evidence provides quantitative data to assess the actual disruption of default modes of coordination in ataxic patients and the possible motor-control gain following rehabilitation therapy. The available experiments show that ataxic patients do not detect the dynamical relationship between environmental features and the motor properties of their own bodies. This means that ataxic patients are unable to judge the scaling of environmental variables in relation to their bodily variables, resulting in the performance of anomalous behavioral patterns.

However, although the study of correlational variables provides a description of the dysfunctional ataxic behavior, this approach offers no cues concerning the causes underlying such conditions. This means that a correlational account can be fruitfully employed to gain information about the variability of the disease symptoms, showing different degrees of severity with respect to standard behavioral patterns, but it cannot be employed for the purpose of etiological diagnosis. Indeed, the methodological tools of DST are not suitable for highlighting the individual causes of a disease phenomenon (see Sect. 3), thus DST is unable to explain why ataxic patients with are impaired in performing visually guided grasping actions. After a complete correlational analysis, one may still require an explanation of why lesions in the parietal cortex correlate with ataxic behaviors, albeit no correlational analysis can answer this question. Though a correlational methodology allows one to predict that lesions in the parietal cortex usually result in the inability of the agent to detect action possibilities in the environment, it seems incompetent in explaining why there are cases in which they reduce errors and relieve their conditions.

Of course, a correlational approach may be able to predict this phenomenon by means of generalizations based on previous cases but is unable to say why such a phenomenon occurs. A correlational account, indeed, is unable to explain why using conceptual information may improve the performance of ataxic patients (Zgaljardic et al., 2011). The mere knowledge that an alteration of cortical parameters is correlated with variations in parameters concerning visually guided actions does not provide sufficient reasons to infer that the recourse to external prostheses (e.g., planners, calendars, recording devices, timers and pagers) in addition to internal cueing (e.g., developing mnemonics or an internal checklist) may reduce errors and relieve

\footnotetext{
${ }^{4}$ Experimental results show that after a measurable lesion in the left posterior parietal cortex, the agent's ability to reach a target is characterized by significant alterations in several parameters such as the initial movement direction, decreased hand velocity, decreased elbow velocity, and increased trajectory curvature (Kamper et al., 2002). A purely correlational analysis also shows that patients with lesions to the parietal cortex have difficulty in performing reaching-to-grasp actions located in the contralesional visual field and with the contralesional hand. In this respect, a relevant discrepancy is observed when ataxic patients use the ataxic hand for actions directed towards the ataxic field, whereas less severe discrepancies are observed when patients use the healthy hand towards the ataxic visual field or the ataxic hand towards the healthy visual field. In contrast, actions performed with the healthy hand towards the healthy visual field exhibit no discrepancies compared with normal subjects (Pisella, Rossetti, \& Rode, 2017).
} 
the condition of patients suffering from optic ataxia (Sect. 4). This means that a rehabilitation program based on such a kind of resource is hardly configurable from the point of view of DST, and its results cannot be explained by means of a correlational approach.

\section{When Computations Explain Better}

Over the last few decades, the dual stream model of visual processing (Jacob \& Jeannerod, 2003; Milner \& Goodale, 1995) served as basic ground to build up a computational architecture according to which an agent computes visuomotor information in the environment (e.g., Cisek \& Kalaska, 2010; Thill et al., 2013; Zipoli Caiani, 2014; Tillas et al., 2017). According to the dual streams model, visual processing involves two subsystems: the dorsal system, which performs processes associated with detecting affordances and visually guiding actions, and the ventral system, which performs processes associated with semantic identification and intentional planning (Goodale \& Milner, 1992).

The essence of the dual streams model of vision lies in the functional differences between the two streams. On one hand, the ventral stream allows an agent to recognize objects in the environment, attaching meanings and establishing causal relations. Such operations are crucial for acquiring a conceptual grasp of the environment, providing resources for incorporating previously stored information into the online control of current actions and making intentional action planning possible (Goodale $\&$ Milner, 1995; Goodale, 2014). On the other hand, the dorsal stream performs transformations that convert information about the shape and location of the source of the stimulus into parameters suitable for action execution. Along the dorsal pathway, the anterior intraparietal area and the ventral premotor cortex extract and compute sensorimotor information from the perceptual stimulus, making it possible to detect action possibilities from the information detected through the retinotopic map (e.g., Andersen \& Buneo, 2003; Mohan et al., 2017; Rizzolatti \& Luppino, 2001). ${ }^{5}$

Importantly, over the last few years, several studies have shown that the ventral stream also biases the detection of action possibilities by exploiting functional interactions with different points of the dorsal processing (Briscoe, 2009; Briscoe \& Schwenkler, 2015; Chinellato \& Pobil, 2016; Zipoli Caiani \& Ferretti, 2017). Among the various interactions between the information processed in the two streams, an

\footnotetext{
${ }^{5} \mathrm{~A}$ generally agreed-upon architecture for affordance perception assumes that visuomotor information is computed by means of a sensorimotor matching mechanism (Rizzolatti \& Sinigaglia, 2008). This amounts to an assumption that action-related information is detected and processed by the agent's sensorimotor apparatus depending on its body shape and motor abilities. According to this view, since the stimulus information in visual perception and the motor information underlying the action are coded together (Prinz, 1997), it seems possible to account for the attentional facilitation that characterizes the detection of action possibilities in terms of visually elicited motor representations (Brozzo, 2017; Butterfill \& Sinigaglia, 2014; Ferretti, 2016, Ferretti \& Zipoli Caiani, 2019).
} 
important connection is precisely that which occurs at the level of the parietal cortex, that is, the region of the brain damaged in optic-ataxic patients. This interaction strongly affects motor preparation and control of movements, suppressing elicited sensorimotor patterns to prevent undesired actions from being triggered. Indeed, information from the ventral stream may help in selecting the relevant patterns of action processed in the dorsal pathway, allowing to the agent's conceptual knowledge to influence the execution of visually guided actions (e.g., Borra et al., 2010; Hoshi \& Tanji, 2007). It may be argued that this computational architecture offers an adaptive advantage to the extent that it allows a fast link between perception, conceptualization and action by means of reliable information integration (Zipoli Caiani, 2018).

Concerning the computational role of the parietal cortex, emerging data from neuropsychology and neuroimaging support the view that portions of this region are devoted to integrating information for guiding actions according to the agent's specific goal (Culham, Cavina-Pratesi, \& Singhal, 2006). Notably, a number of TMS studies have shown that the parietal cortex is functionally involved in the processing of the visual motor information required to adjust the motor plan to perform hand actions and achieve intentional goals (Iacoboni, 2006). Evidence such as this shows that the parietal cortex is responsible for representation and conversion of visual information into movements and for online control of motor actions (Blangero et al., 2010). Lesions in this area, therefore, leave patients without a fundamental structure for visuomotor integration and control, thus causing disorders in the representation of the surrounding objects and impairments in planning and execution of goal-directed actions.

Interestingly, the computational architecture based on the dual stream model of vision explains why patients with lesions to the parietal cortex may suffer from optic ataxia. Moreover, the same architecture explains why ataxic patients exhibit intact performance when a delay is introduced between the perceptual stimulus and the behavioral response or when the patient relies on conceptual knowledge of the target. According to this architecture, the impairment of the parietal cortex does not completely prevent agents from processing and exploiting conceptual information. The massive interaction between the ventral stream and the dorsal stream allows for a reallocation of functions that ensures the recognition of affordances in the environment by means of compensatory strategies such as the exploitation of conceptual cues (Zipoli Caiani \& Ferretti, 2017). This means that, once the functional specializations and reciprocal interactions between the two streams have been defined, it is possible to explain why a lesion in the parietal cortex may induce the inability to immediately detect a pragmatic relation between the agent's body and the features of the environment. Moreover, it is also possible to explain why, in certain circumstances, the use of conceptual information may relieve such a deficit. 


\section{Conclusions}

RE is a view according to which cognitive phenomena should be explained by means of DST instead of a mechanical computational account. Although the adoption of the methodological tools of DST is gaining increasing consensus in the cognitive science of vision, it faces an explanatory shortcoming that should not be underestimated. It is well known, indeed, that descriptive and predictive adequacy do not imply explanatory adequacy (Salmon, 1984). Accordingly, although DST is suitable to provide precise correlations between environmental, bodily and behavioral variables, such a methodology remains silent about the underlying causes of such correlations.

However, by means of a computational architecture based on the dual stream model of vision it has been possible to explain why patients with lesions to the parietal cortex become unable to detect affordances in the environment, as well as why they gain good visuomotor performances given appropriate conditions (see Sect. 6). The computational integration of pragmatic and conceptual information in vision for action, indeed, makes it possible to explain why a lesion in an area of the parietal cortex is correlated with inability to detect and exploit affordances, but also why particular circumstances (e.g., delayed responses and conceptual information) allow the agent to use alternative cognitive strategies to recognize and take advantage of the affordances of the environment.

\section{References}

Andersen, R. A., \& Buneo, C. A. (2003). Sensorimotor integration in posterior parietal cortex. Advances in Neurology, 93, 159-177.

Balint, R. (1909). Seelenlahmung des. Monatsschr Psychiatr Neurol, 25, 51-81.

Bechtel, W., \& Richardson, R. C. (1993). Discovering complexity decomposition and localization as strategies in scientific research. Princeton.

Beer, (2000). Dynamical approaches to cognitive science. Trends in Cognitive Sciences, 4(3), 91-99.

Blangero, A., Ota, H., Rossetti, Y., Fujii, T., Ohtake, H., Tabuchi, M., et al. (2010). Systematic retinotopic reaching error vectors in unilateral optic ataxia. Cortex; a Journal Devoted to the Study of the Nervous System and Behavior, 46(1), 77-93. https://doi.org/10.1016/j.cortex.2009. 02.015 .

Borra, E., Ichinohe, N., Sato, T., Tanifuji, M., \& Rockland, K. S. (2010). Cortical connections to area TE in monkey: hybrid modular and distributed organization. Cerebral Cortex (New York, N.Y.: 1991), 20(2), 257-270. https://doi.org/10.1093/cercor/bhp096.

Briscoe, R. (2009). Egocentric spatial representation in action and perception. Philosophy and Phenomenological Research, 79(2), 423-460.

Briscoe, R., \& Schwenkler, J. (2015). Conscious vision in action. Cognitive Science, 39(7), 14351467.

Brozzo, C. (2017). Motor intentions: how intentions and motor representations come together. Mind Language, 32(2), 231-256. https://doi.org/10.1111/mila.12140.

Bruineberg, J., \& Rietveld, E. (2014). Self-organization, free energy minimization, and optimal grip on a field of affordances. Frontiers in Human Neuroscience, 8, 599. https://doi.org/10.3389/ fnhum.2014.00599. 
Butterfill, S. A., \& Sinigaglia, C. (2014). Intention and motor representation in purposive action: intention and motor representation in purposive action. Philosophy and Phenomenological Research, 88(1), 119-145. https://doi.org/10.1111/j.1933-1592.2012.00604.x.

Cavina-Pratesi, C., Connolly, J. D., \& Milner, A. D. (2013). Optic ataxia as a model to investigate the role of the posterior parietal cortex in visually guided action: evidence from studies of patient M.H. Frontiers in Human Neuroscience, 7. https://doi.org/10.3389/fnhum.2013.00336.

Chemero, A. (2011). Radical embodied cognitive science. Bradford.

Chemero, T., \& Silberstein, M. (2008). After the philosophy of mind: replacing scholasticism with science. Philosophy of Science, 75(1), 1-27.

Chinellato, E., \& del Pobil, A. P. (2016). The neuroscience of action and perception. In The visual neuroscience of robotic grasping (pp. 7-38). Springer, Cham. https://doi.org/10.1007/978-3-31920303-4_2.

Cisek, P., \& Kalaska, J. F. (2010). Neural mechanisms for interacting with a world full of action choices. Annual Review of Neuroscience, 33, 269-298. https://doi.org/10.1146/annurev.neuro. 051508.135409 .

Cohen, M. P. (2015). On Schupbach and Sprenger's measures of explanatory power. Philosophy of Science, 82(1), 97-109. https://doi.org/10.1086/678980.

Colombo, M. (2014). Neural representationalism, the hard problem of content and vitiated verdicts. A reply to Hutto Myin. Phenomenology and the Cognitive Sciences, 13(2), 257-274.

Craver, C. F. (2006). When mechanistic models explain. Synthese, 153(3), 355-376.

Craver, C. F., \& Darden, L. (2013). In search of mechanisms: Discoveries across the life sciences. University of Chicago Press.

Culham, J. C., Cavina-Pratesi, C., \& Singhal, A. (2006). The role of parietal cortex in visuomotor control: what have we learned from neuroimaging? Neuropsychologia, 44(13), 2668-2684. https://doi.org/10.1016/j.neuropsychologia.2005.11.003.

Dennett, D. C. (1987). The intentional stance. MIT Press.

Egan, F. (2013). How to think about mental content. Philosophical Studies, 1, 1-21.

Ferretti, G. (2016). Through the forest of motor representations. Consciousness and Cognition, 43, 177-196. https://doi.org/10.1016/j.concog.2016.05.013.

Ferretti, G., \& Zipoli Caiani, S. (2019). Solving the interface problem without translation: the same format thesis. Pacific Philosophical Quarterly, 100(1), 301-333.

Fodor, J. A., \& Pylyshyn, Z. W. (1988). Connectionism and cognitive architecture. Cognition, 28(1-2), 3-71.

Gallagher, S. (2017). Enactivist interventions: rethinking the mind (1st ed.). Oxford, United Kingdom: Oxford University Press.

van Gelder, T. (1995). What might cognition be if not computation? Journal of Philosophy, 92(7), 345-381.

Gibson, J. J. (1979). The ecological approach to visual perception: Classic edition. Psychology Press.

Goodale, M. A. (2014). How (and why) the visual control of action differs from visual perception. Proceedings. Biological Sciences, 281(1785), 20140337. https://doi.org/10.1098/rspb.2014. 0337.

Goodale, M. A., \& Milner, A. D. (1992). Separate visual pathways for perception and action. Trends in Neurosciences, 15(1), 20-25.

Harrison, H. S., Turvey, M. T., \& Frank, T. D. (2016). Affordance-based perception-action dynamics: A model of visually guided braking. Psychological Review, 123(3), 305-323. https://doi.org/10. 1037/rev0000029.

Heinke, D. (2000). A dynamical system theory approach to cognitive neuroscience. Behavioral and Brain Sciences, 23(4), 543.

Hempel, C. G. (1965). Aspects of scientific explanation: and other essays in the philosophy of science. Free Press. 
Himmelbach, M., \& Karnath, H.-O. (2005). Dorsal and ventral stream interaction: contributions from optic ataxia. Journal of Cognitive Neuroscience, 17(4), 632-640. https://doi.org/10.1162/ 0898929053467514.

Hoshi, E., \& Tanji, J. (2007). Distinctions between dorsal and ventral premotor areas: anatomical connectivity and functional properties. Current Opinion in Neurobiology, 17(2), 234-242. https:// doi.org/10.1016/j.conb.2007.02.003.

Hutto, D. D., Myin, E. (2012). Radicalizing enactivism: Basic minds without content. MIT Press.

Hutto, D. D., Myin, E. (2017). Evolving enactivism: Basic minds meet content. MIT Press. Retrieved from http://www.jstor.org/stable/j.ctt1q1xq5g.

Iacoboni, M. (2006). Visuo-motor integration and control in the human posterior parietal cortex: evidence from TMS and fMRI. Neuropsychologia, 44(13), 2691-2699. https://doi.org/10.1016/ j.neuropsychologia.2006.04.029.

Illari, P., \& Williamson, J. (2012). What is a mechanism? Thinking about mechanisms across the sciences. European Journal for Philosophy of Science, 2(1), 119-135.

Jacob, P., Jeannerod, M. (2003). Ways of seeing: The scope and limits of visual cognition. Oxford University Press.

Kamper, D. G., McKenna-Cole, A. N., Kahn, L. E., \& Reinkensmeyer, D. J. (2002). Alterations in reaching after stroke and their relation to movement direction and impairment severity. Archives of Physical Medicine and Rehabilitation, 83(5), 702-707.

Kaplan, D. M. (2015). Moving parts: The natural alliance between dynamical and mechanistic modeling approaches. Biology and Philosophy, 30(6), 757-786.

Kriegel, U. (2013). The phenomenal intentionality research program. In U. Kriegel (Ed.), Phenomenal intentionality. Oxford University Press.

Lopresti-Goodman, S. M., Turvey, M. T., \& Frank, T. D. (2011). Behavioral dynamics of the affordance "graspable". Attention, Perception, \& Psychophysics, 73(6), 1948-1965. https://doi. org/10.3758/s13414-011-0151-5.

Mark, L. S. (1987). Eyeheight-scaled information about affordances: a study of sitting and stair climbing. Journal of Experimental Psychology. Human Perception and Performance, 13(3), 361370.

Maturana, H. R., \& Varela, F. J. (1991). Autopoiesis and Cognition: The realization of the living. Springer Science \& Business Media.

Mcculloch, W. S., \& Pitts, W. (1944). A logical calculus of the ideas immanent in nervous activity. Journal of Symbolic Logic, 9(2), 49-50.

Milner, A. D., \& Goodale, M. A. (1995). The visual brain in action. Oxford University Press.

Mohan, H., de Haan, R., Mansvelder, H. D., \& de Kock, C. P. J. (2017). The posterior parietal cortex as integrative hub for whisker sensorimotor information. Neuroscience. https://doi.org/10.1016/ j.neuroscience.2017.06.020.

Noë, A. (2004). Action in perception. MIT Press.

O'Regan, J. K. (2011). Why red doesn't sound like a bell: Understanding the feel of consciousness. Oxford University Press.

O'Regan, J. K., \& Noë, A. (2001). A sensorimotor account of vision and visual consciousness. The Behavioral and Brain Sciences, 24(5), 939-973; discussion 973-1031.

Paolo, E. D. (2009). Extended life. Topoi, 28(1), 9-21.

Pietroski, P. M. (1992). Intentionality and teleological error. Pacific Philosophical Quarterly, 73(3), 267-282.

Pisella, L., Gréa, H., Tilikete, C., Vighetto, A., Desmurget, M., Rode, G., et al. (2000). An “automatic pilot" for the hand in human posterior parietal cortex: toward reinterpreting optic ataxia. Nature Neuroscience, 3(7), 729-736. https://doi.org/10.1038/76694.

Pisella, L., Rossetti, Y., \& Rode, G. (2017). Optic ataxia in Bálint-Holmes syndrome. Annals of Physical and Rehabilitation Medicine, 60(3), 148-154. https://doi.org/10.1016/j.rehab.2016. 01.003 .

Prinz, W. (1997). Perception and action planning. European Journal of Cognitive Psychology, 9(2), 129-154. https://doi.org/10.1080/713752551. 
Putnam, H. (1967). Psychological predicates. In W. H. Capitan \& D. D. Merrill (Eds.), Art, mind, and religion (pp. 37-48). University of Pittsburgh Press.

Ramsey, W. (2015). Must cognition be representational? Synthese. https://doi.org/10.1007/s11229014-0644-6.

Ramsey, W. M. (2007). Representation reconsidered. Cambridge University Press.

Rietveld, E., \& Kiverstein, J. (2014). A rich landscape of affordances. Ecological Psychology, 26(4), 325-352. https://doi.org/10.1080/10407413.2014.958035.

Rizzolatti, G., \& Luppino, G. (2001). The cortical motor system. Neuron, 31(6), 889-901.

Rizzolatti, G., \& Sinigaglia, C. (2008). Mirrors in the brain: How our minds share actions and emotions. Oxford University Press.

Salmon, W. (1984). Scientific explanation and the causal structure of the world. Princeton University Press.

Schindler, I., Rice, N. J., McIntosh, R. D., Rossetti, Y., Vighetto, A., \& Milner, A. D. (2004). Automatic avoidance of obstacles is a dorsal stream function: evidence from optic ataxia. Nature Neuroscience, 7(7), 779-784. https://doi.org/10.1038/nn1273.

Schupbach, J. N., \& Sprenger, J. (2011). The Logic of Explanatory Power*. Philosophy of Science, 78(1), 105-127. https://doi.org/10.1086/658111.

Spivey, M. (2008). The continuity of mind. Oxford, New York: Oxford University Press.

Thelen, E., \& Smith, L. B. (1996). A dynamic systems approach to the development of cognition and action. MIT Press.

Thill, S., Caligiore, D., Borghi, A. M., Ziemke, T., \& Baldassarre, G. (2013). Theories and computational models of affordance and mirror systems: an integrative review. Neuroscience and Biobehavioral Reviews, 37(3), 491-521. https://doi.org/10.1016/j.neubiorev.2013.01.012.

Thompson, E. (2007). Mind in life: Biology, phenomenology, and the sciences of mind. Harvard University Press.

Tillas, A., Vosgerau, G., Seuchter, T., \& Zipoli Caiani, S. (2017). Can affordances explain behavior? Review of Philosophy and Psychology, 8(2), 295-315.

Varela, F., Thompson, E., \& Rosch, E. (1991). The embodied mind: Cognitive science and human experience. MIT Press.

Walmsley, J. (2008). Explanation in dynamical cognitive science. Minds and Machines, 18(3), 331-348.

Zgaljardic, D. J., Yancy, S., Levinson, J., Morales, G., \& Masel, B. E. (2011). Balint's syndrome and post-acute brain injury rehabilitation: a case report. Brain Injury, 25(9), 909-917. https://doi. org/10.3109/02699052.2011.585506.

Zipoli Caiani, S., \& Ferretti, G. (2017). Semantic and pragmatic integration in vision for action. Consciousness and Cognition, 48, 40-54. https://doi.org/10.1016/j.concog.2016.10.009.

Zipoli Caiani, S. (2014). Extending the notion of affordance. Phenomenology and the Cognitive Sciences, 13(2), 275-293. https://doi.org/10.1007/s11097-013-9295-1.

Zipoli Caiani, S. (2018). Intensional biases in affordance perception. Synthese. https://doi.org/10. 1007/s11229-018-02049-. 
Open Access This chapter is licensed under the terms of the Creative Commons Attribution 4.0 International License (http://creativecommons.org/licenses/by/4.0/), which permits use, sharing, adaptation, distribution and reproduction in any medium or format, as long as you give appropriate credit to the original author(s) and the source, provide a link to the Creative Commons license and indicate if changes were made.

The images or other third party material in this chapter are included in the chapter's Creative Commons license, unless indicated otherwise in a credit line to the material. If material is not included in the chapter's Creative Commons license and your intended use is not permitted by statutory regulation or exceeds the permitted use, you will need to obtain permission directly from the copyright holder.

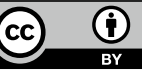

